\title{
Un proyecto educativo común: la relación familia y escuela. Revisión de investigaciones y normativas
}

\section{A shared educational project: the family and school relationship. Review of research and regulations}

\author{
Noelia Ceballos López ${ }^{1}$ \\ ceballosn@unican.es \\ ÁnGela SAIZ LiNARES \\ saizla@unican.es \\ Universidad de Cantabria, Santander, España
}

\section{Resumen:}

Este trabajo tiene como foco de indagación los procesos de participación familiar en las escuelas de educación infantil y primaria. Para ello, se ha realizado una revisión bibliográfica y normativa bajo un diseño de carácter cualitativo de revisión sistemática de la literatura (análisis de contenido). De dicho análisis emerge una cartografía de la relación familia-escuela en torno a tres ejes: los procesos de enseñanza-aprendizaje, la atención a la diversidad; y la participación de las familias en la organización y gestión de la escuela. Finalmente, enunciamos algunas reflexiones que buscan contribuir a la reflexión sobre la relación familia-escuela y encaminar significados y actuaciones comprometidas con los objetivos inclusivos y democráticos.

\section{Palabras clave:}

Relación familia-escuela; revisión bibliográfica; escuela democrática; participación de las familias.

\begin{abstract}
:
This study aims to explore the processes of family participation in infant and primary schools. For this purpose, we conducted a bibliographic and normative systematic review which adopted a qualitative design based on the analysis of content. From this analysis, a cartography of the familyschool relationship emerges around three pillars: teaching-learning processes, diversity and education, and participation of families in the school organization and management. Finally, we formulate some conclusions that seek to contribute to the reflection on the family-school relationship and to highlight meanings and actions which follow inclusive and democratic principles.
\end{abstract}

\section{Key words:}

Family-school relationship; literature review; democratic school; family's participation.

1 Dirección para correspondencia (correspondence address):

Noelia Ceballos López. Departamento de Educación. Universidad de Cantabria. Av. de los castros s/n. Facultad de Educación, CP. 39005, Santander, Cantabria (España). 
Un proyecto educativo común: la relación familia y escuela. Revisión de investigaciones y normativas

Noelia Ceballos lópez y Ángela Saiz Linares

\section{Résumé:}

Ce travail se concentre sur les processus de participation dans les écoles maternelles et primaires. Pour cela, une revue bibliographique et normative a été réalisée dans le cadre d'une conception qualitative d'une revue systématique de la littérature (analyse de contenu). De cette analyse émerge une cartographie de la relation famille-école autour de trois axes: les processus d'enseignement-apprentissage, I'attention à la diversité, et la participation des familles dans l'organisation et dans la gestion de l'école. Finalement, nous formulerons des réflexions visant à contribuer à la réflexion sur la relation familleécole et à orienter les significations et les actions engagées vers les objectifs inclusifs et démocratiques.

\section{Mots clés:}

Relation famille-école; révision bibliographique; école démocratique; participation des familles.

Fecha de recepción: 11-05-2018

Fecha de aceptación: 04-07-2019

\section{Introducción}

La investigación sobre participación escolar ha evidenciado la importancia de la implicación de las familias en la vida de las escuelas (Castro y García, 2016; Epstein, 2001; Hernández y López, 2006). El Informe Europeo sobre Calidad de la Educación Escolar estima que la colaboración de las familias es un índice de calidad al influir en el aprendizaje del alumnado, su desarrollo social y personal (Comisión Europea, 2000). Una idea que es reforzada por estudios empíricos recientes (Issó, Becerril y Venegas, 2019; Kim y Bryan, 2017; Reininger y Santana López, 2017; Saracostti, Lara, Martella, Miranda, Miranda-Zapata y Reininger, 2019). En este trabajo nos aproximamos al concepto de relación familiaescuela desde la revisión de diferentes estudios, investigaciones y legislaciones nacionales. Este estudio forma parte de una investigación más amplia destinada a analizar y profundizar, desde una perspectiva crítico-reflexiva, las relaciones familia-escuela en los centros educativos de Educación Infantil y Primaria documentadas por los maestros en formación de la Universidad de Cantabria en el marco del Practicum (Ceballos y Saiz, en prensa). Si bien existe consenso sobre el valor de dicha participación, encontramos diversas formas de conceptualizarla y de promoverla. En la siguiente tabla 1, sintetizamos algunas propuestas de conceptualización realizadas por diversos autores. 
Tabla 1

Formas de concebir la participación de las familias por diferentes autores

\begin{tabular}{|c|c|}
\hline Autores & Tipo de participación de las familias \\
\hline $\begin{array}{l}\text { Pugh y De'Ath } \\
\text { (1989) }\end{array}$ & $\begin{array}{c}\text { Activos en el gobierno de la escuela } \\
\text { Ayudan en las sesiones en el centro } \\
\text { Trabajan con el personal de modo habitual } \\
\text { Colaboran en la toma de decisiones sobre la labor docente }\end{array}$ \\
\hline $\begin{array}{l}\text { Eccles y Harold } \\
\text { (1996) }\end{array}$ & $\begin{array}{c}\text { Monitorización } \\
\text { Voluntariado } \\
\text { Implicación } \\
\text { Contacto de la familia para informar sobre el progreso } \\
\text { educativo } \\
\text { Ayuda puntual }\end{array}$ \\
\hline Epstein (2001) & $\begin{array}{c}\text { Ser padres } \\
\text { Voluntariado } \\
\text { Comunicación } \\
\text { Toma de decisiones } \\
\text { Colaborar en casa } \\
\text { Colaborar con la comunidad } \\
\end{array}$ \\
\hline $\begin{array}{l}\text { Kohl, Lengua } \\
\text { y Mcmahom } \\
\quad(2000)\end{array}$ & $\begin{array}{c}\text { La participación en el entorno escolar } \\
\text { Realización de tareas en casa } \\
\text { Apoyo a la escuela } \\
\text { Contacto entre padres y educadores } \\
\text { La calidad de la relación entre padres y profesores } \\
\text { La percepción de los profesores sobre el rol que ejercen los } \\
\text { padres. }\end{array}$ \\
\hline $\begin{array}{c}\text { Bastiani (1987) } \\
\text { Macbeth (1984) } \\
\text { Paterman (1970) } \\
\text { García-Bacete } \\
\text { (2003) }\end{array}$ & $\begin{array}{c}\text { Participación informativa o pseudoparticipación } \\
\text { Participación consultiva } \\
\text { Participación en planes o proyectos del centro }\end{array}$ \\
\hline $\begin{array}{l}\text { Auduc }(2007) \\
\text { Garreta }(2013)\end{array}$ & $\begin{array}{c}\text { Participación individual: asistiendo a las reuniones, segui- } \\
\text { miento escolar de sus hijos, etc. } \\
\text { Participación colectiva: AMPA y Consejo Escolar }\end{array}$ \\
\hline Maestre (2009) & $\begin{array}{c}\text { Vinculada con el aula } \\
\text { Vinculada con el centro educativo }\end{array}$ \\
\hline $\begin{array}{l}\text { Arostegui, } \\
\text { Darretxe y } \\
\text { Beloki (2013) }\end{array}$ & $\begin{array}{c}\text { Participación informativa } \\
\text { Participación consultiva } \\
\text { Participación decisoria } \\
\text { Participación evaluativa } \\
\text { Participación educativa en el aprendizaje de los niños y } \\
\text { niñas y en su propio aprendizaje }\end{array}$ \\
\hline
\end{tabular}




\begin{tabular}{cc}
\hline Autores & Tipo de participación de las familias \\
\hline $\begin{array}{c}\text { Llevot y Bernad } \\
(2015)\end{array}$ & $\begin{array}{c}\text { Formal: asistencia a las reuniones de inicio de curso, etc. } \\
\text { Informal: conversaciones a las entradas y salidas de la } \\
\text { escuela, etc. }\end{array}$ \\
\hline
\end{tabular}

Por un lado, proponemos que la participación de las familias sea analizada en función de su alcance: en un polo, aparece restringida a asuntos individuales que atañen únicamente a un estudiante; y, en el otro, encontramos propuestas de corte colectivo vinculadas al aula y al centro educativo (Auduc, 2007; García-Bacete, 2003; Garreta, 2013).

Por otro lado, proponemos sintetizar las diversas opciones presentadas en la tabla 1 en cuatro grados de participación de menor a mayor complejidad. En un primer nivel, encontramos propuestas en que las familias asumen un rol receptivo y de emisión de información en espacios formales (reuniones, entrevistas, circulares, etc.) e informales (entradas y salidas) (Llevot y Bernad, 2015; Méndez, Ruiz, Rodríguez y Rebaque, 2010).

Un segundo nivel, define la implicación de las familias como agentes consultados, especialmente referido a las medidas de atención a la diversidad. Como señala la normativa, las familias deben ser informadas y escuchadas en el proceso de promoción e incorporación de medidas, aunque no se garantiza que su opinión sea considerada en la toma de decisiones.

En una tercera forma, las familias son invitadas a involucrarse en actividades puntuales habitualmente pautadas por el centro: la maleta viajera, "ir de visita a clase a...", etc.

Finalmente, surgen propuestas donde las familias asumen un papel protagónico en el proceso de enseñanza-aprendizaje y en la organización y gestión de la escuela. Así, la vida de la escuela se caracteriza por su carácter eminentemente colaborativo donde familias, alumnado y docentes comparten metas y toman decisiones sustentadas en el bien común (García-Albaladejo, 2006; Llevot y Bernad, 2015). Es esta última opción la que se acerca a la concepción de participación más compleja, entendida como la capacidad de formar parte de los procesos de planificación, toma de decisiones, acción y evaluación de la vida del aula y del centro (Susinos y Ceballos, 2012). 


\section{Metodología}

El objetivo del artículo es cartografiar la participación de la familia en centros de educación infantil y primaria a través de ejes de acción que surgen de la revisión y análisis de estudios teóricos, investigaciones empíricas y normativas. Optamos por un diseño de carácter cualitativo de revisión sistemática de la literatura (Creswell, 1994), con la finalidad de compendiar el conocimiento existente sobre nuestro tema de estudio. Pretendemos construir una propuesta que permita a los centros reflexionar sobre cómo responden a este espacio de la vida escolar, así como imaginar otras acciones posibles.

Nos planteamos preguntas como: ¿cómo se entiende la relación de las familias con la escuela? ¿Cómo se define su implicación en los procesos de enseñanza-aprendizaje? ¿Y en los asuntos propios de la atención a la diversidad? ¿Cómo se define y articula su inclusión en la vida de la escuela? ¿Qué canales de participación se utilizan?

Para responder a estos interrogantes, se han analizado artículos, informes de investigación y libros que abordan epistemológicamente nuestro tema de interés. Los criterios de selección de las fuentes de información son: en relación a los artículos, publicados a partir del 2000, se recurrió a la base datos SCOPUS, mientras que los libros fueron consultados en la base de datos de libros editados en España. Se priorizaron obras enmarcadas en la Acción Tutorial, por ser el marco de trabajo de los docentes en las relaciones familia-escuela (Longás y Mollá, 2007). En ambos casos, la búsqueda se realizó atendiendo a las claves: "participación" "relación" "implicación" "acción tutorial" combinadas con "familia y escuela" "padres" "familia".

En un segundo momento selectivo, las referencias se seleccionaron atendiendo a su pertinencia para responder a las preguntas de investigación.

Por otra parte, se ha recurrido al análisis de referencias normativas de diverso rango siguiendo el criterio de pertinencia, es decir, normativa que actualmente se encuentra en vigor:

- LOMCE (2013). Normativa nacional para todo el sistema educativo.

- Normativa de la Comunidad Autónoma de Cantabria:

o Decretos y Órdenes que regulan la organización, funcionamiento y gestión de los centros de Educación Infantil y Primaria.

o Decretos y Órdenes propias de la etapa de Educación Infantil. 
o Decretos y Órdenes propias de la etapa de Educación Primaria.

o Decretos y Órdenes relativos a la orientación y atención a la diversidad.

Para el tratamiento de la información, realizamos un análisis de contenido (López-Noguero, 2002; Mendes y Miskulin, 2017) que busca acceder de manera sistemática a tres niveles de análisis: descriptivo, tratando de identificar las afirmaciones, ideas y propuestas del discurso presente; analítico, organizando las formulaciones atendiendo a las categorías de análisis definidas; $y$, por último, interpretativo, que permite comprender la información y dotarla de sentido (Ruiz-Silva, 2004).

\section{Resultados}

\section{Una mirada a la normativa sobre la relación familia-escuela}

Este apartado recoge las principales reflexiones acerca del sentido, funciones y estrategias encaminadas a promover la relación familia-escuela en la normativa, comenzando con la vigente Ley educativa para, posteriormente, analizar las diferentes normativas de Cantabria.

En la LOMCE encontramos una contradicción entre el discurso explícito del preámbulo y las interpretaciones que emergen de las acciones definidas en los diversos artículos. Esta introducción aprecia a la familia como un elemento favorecedor del desarrollo del alumnado "necesario para asegurar una educación de calidad con equidad" (LOMCE). En tal sentido, solicita a los centros la generación espacios y canales de trabajo conjunto.

A fin de hacer efectiva la corresponsabilidad entre el profesorado y las familias en la educación de sus hijos, las Administraciones educativas adoptarán medidas que promuevan e incentiven la colaboración efectiva entre la familia y la escuela (LOMCE).

Sin embargo, también asoman algunas referencias que limitan dicha implicación. Por ejemplo, cuando apela a la responsabilidad de las empresas en la educación de los niños, entrelazando un discurso de participación democrática de las familias y la ciudadanía con un posi- 
cionamiento más propio de un sistema mercantilista, profesionalizador y altamente selectivo.

La educación es una tarea que afecta a empresas, asociaciones, sindicatos, organizaciones no gubernamentales, así como a cualquier otra forma de manifestación de la sociedad civil y, de manera muy particular, a las familias (LOMCE)

Asimismo, prevalece el intercambio de información como acción predominante para favorecer la colaboración familia-escuela.

La información periódica a las familias sobre el proceso de aprendizaje de sus hijos e hijas, así como la orientación para su cooperación en el mismo [profesorado] (LOMCE)

En menores ocasiones se definen canales de colaboración e implicación directa en la organización y gestión educativa del centro, principalmente encaminados a la presencia de las familias en los órganos de gobierno (AMPA y Consejo escolar), donde desarrollan actividades paralelas al currículo (actividades extraescolares, etc.) o apoyos ligados al aumento del rendimiento académico:

Aprobar [...] las aportaciones de las familias de los alumnos y alumnas para la realización de actividades extraescolares y los servicios escolares cuando así lo hayan determinado las Administraciones educativas [funciones del consejo escolar] (LOMCE)

- Normativa sobre la organización y gestión de centros educativos de Educación Infantil y Primaria de Cantabria

La principal reflexión que emerge de esta normativa es el mayor peso otorgado al intercambio de información, esencialmente desde el centro a la familia, identificando con precisión ámbitos y tipos de información: faltas de asistencia y puntualidad; incidencias con el alumnado, actividades docentes, proceso de enseñanza y aprendizaje, procedimientos e instrumentos previstos para la evaluación continua del aprendizaje, progreso educativo, condiciones de promoción, plan de convivencia, normas de organización y funcionamiento del mismo o actividades comple- 
mentarias y extraescolares. Estos ámbitos relegan a las familias a un rol receptivo de información seleccionada por los docentes.

Complementariamente, se definen los espacios destinados a dicha comunicación (reuniones, entrevistas, etc.):

Al principio del curso, los tutores celebrarán una reunión con el conjunto de los padres, madres o representantes legales de los grupos del mismo curso [...] (Orden EDU/65/2010)

En contraposición, se hace una breve referencia a la colaboración, pero en términos intencionales y no concretando pautas de acción:

Facilitar la colaboración entre los profesores y los padres, madres o representantes legales de los alumnos (Decreto 25/2010)

- Normativa que regula el currículo de las etapas de Educación Infantil y Primaria

Igualmente, en el análisis de la normativa que busca definir el currículum de las etapas de Educación Infantil y Primaria, las principales actuaciones se circunscriben a noticiar a las familias. Por un lado, sobre el progreso educativo y evaluación del alumnado en un intercambio unidireccional desde el centro hacia la familia.

Dicha cooperación incluirá la comunicación periódica por medio de informes escritos, entrevistas individuales y reuniones colectivas (Orden EDU/105/2008)

Por otro, sobre el funcionamiento del aula y del centro y las características principales de la propuesta educativa, con un carácter eminentemente informativo y no como oportunidad de reflexión y diálogo conjunto.

Al comienzo del curso escolar, el equipo docente, coordinado por el tutor, elaborará por escrito la información relativa a la programación didáctica, que dará a conocer a los alumnos y a sus familias a través del tutor.

Se manifiesta la excepción durante el período de acogida, en el que 
se definen momentos destinados a demandar información a las familias.

Al incorporarse por primera vez un alumno a un centro de Educación Infantil, el maestro tutor realizará una evaluación inicial que recogerá los datos más destacados de su desarrollo, tendrá en cuenta los datos relevantes aportados por la familia en la entrevista inicial [...]. (Orden EDU/105/2008)

Es destacable su papel como informadores clave en este proceso de acogida pues, posteriormente, la información se define en las normativas como un proceso unidireccional que va de la escuela a la familia, silenciando la información que en el ámbito familiar se produce sobre la escuela.

- Normativa de atención a la diversidad en centros educativos de Educación Infantil y Primaria

No es nuestro propósito realizar un análisis en profundidad sobre la normativa de la atención a la diversidad. Pretendemos reflexionar sobre las acciones que desde el marco de la orientación educativa y, más específicamente, de la normativa de atención a la diversidad, se definen en la relación familia-escuela con una mirada dirigida al trabajo de los tutores.

Con dicha especificidad, hallamos una participación de las familias restringida al intercambio de información y circunscrita principalmente a las familias de alumnado con medidas de atención a la diversidad. Principalmente, encontramos diferentes acciones encaminadas a: el consentimiento informado y autorización de las familias para la elaboración del informe y el desarrollo de medidas específicas o extraordinarias de atención a la diversidad.

Solicitar autorización para la aplicación de medidas de atención a la diversidad (EDU/21/2006).

El consentimiento informado implica que las familias tienen conocimiento suficiente para comprender el sentido e implicaciones de dichas medidas. Sin embargo, la normativa no profundiza sobre cómo debe producirse o qué implica el consentimiento informado.

Asimismo, se define como función del centro informar y aportar información a las familias sobre el PAD, aunque no se reconoce el diálogo y 
negociación de las medidas con las familias. La excepción se encuentra en las decisiones vinculadas a la permanencia/promoción del alumnado. Aunque la decisión recae en el tutor, esta debe sustentarse en la información recogida del equipo docente y de la familia (Decreto 98/2005). En resumen, la implicación de las familias es más simbólica que real, esto es, son escuchados, pero se desconsidera el poder de influir de manera significativa en la decisión.

\section{Una mirada a una selección de estudios publicados sobre la relación familia-escuela}

Del análisis de los estudios sobre la relación familia-escuela destacamos tres acciones predominantes: el intercambio de información; la colaboración en los procesos de enseñanza-aprendizaje; y la participación en la vida del centro.

- Intercambio de información, comunicación y relaciones

Al igual que en la normativa, estas actuaciones se sitúan como ejes preferentes en la relación familia-escuela, aunque encontramos diversas aproximaciones. De un lado, surgen propuestas focalizadas en la unidireccionalidad de la información desde la escuela a la familia, predominando la relativa a la actividad docente y al rendimiento académico del alumnado (Méndez, Ruiz, Rodríguez y Rebaque, 2010).

Asimismo, prima la información relativa a asuntos que afecten de manera directa a sus hijos (Cánovas y Sahuquillo, 2012; Pastor, 2009), priorizando las situaciones individualizadas y relegando la corresponsabilidad más amplia con el centro educativo.

También se recoge la función asesora de la escuela sobre asuntos académicos, de promoción, familiar o personal, situando la relación entre maestros y familia en un marco de poder desigual entre quienes son expertos (maestros) y quienes asumen las actuaciones que estos definen (familias).

Asesoramiento individual y en pequeño grupo (counseling) con los diferentes agentes de intervención sobre contenidos de tipo personal, familiar, escolar, profesional, laboral, social, problemáticas interpersonales, etc. con un carácter preventivo, de desarrollo y de intervención social (Álvarez, 2017). 
En contraposición, Boza, Salas, Ipland, Aguaded y Fondón (2005), Angulo (2009) o Pantoja (2013), proponen abrir canales de comunicación bidireccionales, donde las familias sean agentes de diálogo con el equipo docente.

- Colaboración en los procesos de aprendizaje

Una segunda línea se vincula al establecimiento de canales de colaboración entre docentes y familias, especialmente a través del tutor, quien se sitúa como figura de referencia y coordinación entre el equipo docente y las familias (Méndez, Ruiz, Rodríguez y Rebaque, 2010; Pantoja, 2013).

Se describen tres objetos de colaboración: el acompañamiento por parte de las familias en el aprendizaje del alumnado, su implicación en la toma de decisiones sobre su progreso/permanencia y el establecimiento de pautas comunes que supongan un apoyo al aprendizaje del alumnado (Boza et al., 2005; Cánovas y Sahuquillo, 2012; Pastor, 2009) y que también pueden exceder lo académico. Para ello, se sugiere la construcción de espacios ad hoc de diálogo.

En un segundo espacio, surgen propuestas encaminadas a la colaboración con las familias en la toma de decisiones sobre los procesos de permanencia o promoción (Méndez, Ruiz, Rodríguez y Rebaque, 2010). Como se destacó en el análisis normativo, las familias deben ser escuchadas antes de tomar la decisión, aunque esta recae en el tutor/a y equipo docente.

Excepcionalmente aparecen algunas vías de colaboración que buscan ampliar los márgenes de acción y superan el apoyo al aprendizaje instrumental, reconociendo los beneficios de la colaboración familiaescuela para el desarrollo personal, académico, cívico y social del alumnado (Cánovas y Sahuquillo, 2012).

- Presencia y participación en la vida del centro

Finalmente, encontramos una invitación (Pantoja, 2013; Urosa y Lázaro, 2017) a fomentar una participación democrática de las familias en la escuela "facilitando situaciones y cauces de comunicación y colaboración y promoviendo la presencia y participación en la vida de los centros" (Pantoja, 2013). 
Si bien no profundizan en el tipo de participación (delegada, directa, deliberativa, etc.), se sitúa la propuesta como una palanca de cambio al invitar a las escuelas a repensar los espacios de presencia y poder de decisión de las familias, los asuntos en los que están implicados, así como su papel y grado de participación.

\section{Discusión de los resultados: Cartografía de la relación familia- escuela}

Esta sección tiene la intención de abrir el debate y proponer nuevos hilos de pensamiento en torno a tres grandes ejes de acción: los procesos de enseñanza-aprendizaje, la atención a la diversidad, y la participación y la convivencia.

\section{La relación familias-tutor: es imprescindible transitar del "estar informado" a "ser parte"}

Focalizar la relación familia-escuela en el intercambio de información, y principalmente en la evaluación, devuelve una idea de educación caracterizada por su instrumentalismo y una concepción del currículum como contenido (Angulo, 1994). Esto es, limitada a la trasmisión y reproducción de la cultura disciplinar y la selección de los mejores estudiantes, aquellos que se adaptan en mayor grado a las medidas y requerimientos académicos. En consecuencia, las familias no son consideradas agentes educativos relevantes, sino sujetos informados sobre el progreso y resultados de sus hijos.

Si bien el intercambio de información, entendido como el ejercicio del derecho de las familias a conocer el desarrollo de sus hijos, es importante, resulta urgente avanzar hacia su consideración como paso indispensable para promover la relación y la participación democrática (Macià, 2016; Sarramona y Rodríguez, 2010). Esto implica que las familias accedan a un conocimiento real de la vida escolar presentado de forma clara y accesible, sin términos y códigos educativos que dificultan el entendimiento e invisibilizan lo que acontece en la escuela. Además, involucra el intercambio recíproco, la posibilidad de dialogar, problematizar, argumentar y avanzar juntos (López, Ridao y Sánchez, 2004). Quizás esta segunda acción es la menos desarrollada en las escuelas. 
Respecto a los espacios de intercambio de información, Garreta (2015) destaca como habituales las reuniones de inicio o final de curso, las entrevistas personales con las familias, la agenda escolar y las circulares y notas. Aunque inicialmente estos espacios son lugares que permiten el diálogo, en la práctica se configuran como instrumentos de intercambio unidireccional de información (docente-familia) o, en su caso, como espacios de requerimiento de información a la familia, altamente pautada y con un propósito definido previamente por el docente. Asimismo, el desarrollo de las nuevas tecnologías se presenta como una oportunidad para abrir nuevos espacios de comunicación. El blog de aula, la web del centro, las plataformas digitales o el correo electrónico (Ocampo, Caeiro y Sarmiento, 2012) son instrumentos habituales en las escuelas, cuya utilización responde a fines informativos, ilustrativos o descriptivos desde el centro a las familias (Macià, 2016). Sin embargo, podemos ampliar esta función y utilizarlos como canales de comunicación y participación de las familias en la escuela (Aguilar y Leiva, 2012).

Asimismo, sería necesario ampliar el foco hacia una implicación en el currículum como elemento clave de la vida escolar. Una escuela con una cultura democrática, además de favorecer el intercambio de información y la participación de todos sus miembros en los aspectos organizativos y de gestión, debe promover un currículum democrático donde se negocian los objetivos, las actividades, los modos de evaluación y cualquier otro elemento curricular (Apple y Beane, 2005). Esto hace referencia a un currículum como realidad interactiva (Angulo, 1994), como una construcción realizada entre los docentes y los alumnos y, en general, de todos aquellos que directa o indirectamente participan en la escuela. En tal sentido, la colaboración con las familias puede convertirse en una excelente palanca de reconocimiento y justicia con aquellas realidades (culturales, económicos, sociales, personales, familiares, etc.) que han sido tradicionalmente olvidadas o discriminadas en la escuela.

Sin lugar a dudas esta es una de las acciones que requieren nuestra atención, pues las experiencias en las que las familias se convierten en interlocutoras en torno a qué y cómo se aprende en la escuela son escasas, aunque encontramos algunos ejemplos evocadores (Álvarez, Campos, Carmona, Magan, Martínez y Sánchez, 2014; Proyecto Atlántida, 2003). 


\section{La relación familia-escuela: de una escuela que tolera mal las diferencias a una escuela para todos.}

La escuela se ha presentado tradicionalmente como una institución que tolera mal las diferencias desarrollando prácticas homogeneizadoras que categorizan y ordenan unos aprendizajes que son idénticos para todos los estudiantes. Del mismo modo, ha favorecido que aquellas familias que no se identifican con la cultura escolar sean poco reconocidas o desplazadas (Garreta, 2010). Por ello, es necesario ampliar el foco a una escuela que defiende los principios de la inclusión educativa y al desarrollo de políticas y prácticas que permiten a todo el alumnado y sus familias, sin excepción, tener presencia, participación y beneficiarse de sus experiencias educativas.

Construir una cultura educativa inclusiva supone transformar la cultura de la escuela en su conjunto (Slee, 2011), sus relaciones, prácticas, curriculum, las relaciones de poder y roles pensando en todo el alumnado y sus familias. En este proceso las familias se sitúan como agentes de análisis de lo acontece, de los procesos de aprendizaje de sus hijos, de sus dificultades y de aquellos aspectos que les ayudan a avanzar. De este modo, todas las familias, pero especialmente aquellas cuyos hijos son habitualmente silenciados en las prácticas escolares, se convierten en testigos expertos que pueden ofrecer una mirada privilegiada, singular y única sobre la vida escolar.

Además, diferentes autores señalan la diversidad familiar existente (Bolívar, 2006; Corpas, Ramírez, Mérida y González, 2012) y apremian a reconocer que no existe una voz homogénea en las familias. Debemos cuestionarnos si los espacios escolares acogen a todas las familias o si, por el contrario, alejan a aquellas que no se identifican con los discursos, términos y modos de organizarse habituales.

\section{La relación familias-tutor: avanzar de la participación simbólica actual a la participación democrática en la vida de la escuela.}

Del análisis realizado se desprende una idea de participación ligada a la acción representativa, informativa y puntual, definiendo unos espacios para dichos fines:

- Reuniones grupales y entrevistas personales 
Estos espacios, definidos como oportunidades de encuentro personal, se caracterizan por ser poco atractivos y materialmente poco relevantes para favorecer la colaboración (Besalú, 2010). Es necesario, por tanto, abordar la acogida a las familias y los estudiantes en términos diferentes a los propuestos. Estos espacios se convierten en lugares de transvase de información estereotipada, normativa o sobre aspectos extracurriculares. Como señala Besalú (2010), esto ahonda en una aproximación al proyecto educativo por parte de las familias escasa y parcial. Por ello, en el momento de la acogida, y probablemente durante toda la escolaridad, los docentes deben compartir la información con las familias para poder someter a consideración y diálogo las acciones emprendidas y pensar las alternativas posibles.

Si bien las investigaciones y normativas señalan la relevancia de la implicación de las familias, la realidad de las escuelas lo que evidencia es desconcierto y desafección. Los docentes consideran que las familias no hacen uso de los espacios de implicación que estos ofrecen. Mientras, las familias consideran que los centros educativos son lugares desconocidos a los que es difícil acceder más allá de lo que ellos quieren mostrar. Por ello se hace imprescindible abordar la participación democrática en la vida de la escuela de las familias, emergiendo cuestiones acerca de: ¿Cómo implicamos a las familias en la vida de la escuela de sus hijos? ¿Cuál es su rol en la convivencia escolar? ¿Qué espacios tienen de participación y sobre qué aspectos?

\section{- El Consejo Escolar y las AMPAS}

En ocasiones, se hace referencia a la participación formal, aquella que tiene lugar en estructuras y órganos normativamente definidos, especialmente: el Consejo escolar y las asociaciones de familias (Egido, 2014).

El número de familias que pertenecen a las asociaciones de familias (AMPA) se ha reducido en los últimos años, siendo aún más escasa su implicación real en las actividades de la asociación (Feito, 2014; Fernández-Enguita, 1993; Garreta, 2008; Giró, Mata, Vallespir y Vigo, 2014). Por otro lado, como señala Mata (2016), las familias tienden a identificar estos espacios como lugares burocratizados, en cuyas reuniones únicamente se aporta información general y se tramitan los aspectos económicos. Asimismo, estos espacios aparecen gestionados por un re- 
ducido grupo de familias que canalizan las acciones, o son lugares con funciones secundarias con poca incidencia en las decisiones del centro.

Por su parte, encontramos que la participación de las familias en la elección de aquellos que les representan en el Consejo Escolar es escasa (Consejo Escolar del Estado, 2014) y diverge a lo largo de las etapas educativas:

Muchos progenitores participan más a nivel colectivo cuando los hijos son pequeños, participando en los órganos establecidos y también colaborando en actividades del centro y del AMPA. En cambio, esta implicación se centra más en el seguimiento escolar de los hijos a medida que éstos crecen. (Llevot y Bernad, 2015, p.67)

Estas diferencias pueden explicarse por cierto desconocimiento de estos órganos (Fernández-Prada, 2003; Llevot y Bernad, 2015) o, puede tener como causa una perspectiva democrática y comunitaria que oculta una prevalencia del individualismo ("únicamente si afecta a mi hijo") (Santos-Guerra, 2000). Sin embargo, hay una consideración que nos interpela a los maestros: la presencia de una participación de las familias más simbólica que real (Feito, 2014) que provoca desafección de las familias con los espacios de participación. Los Consejos Escolares se configuran, por parte de los centros, como reuniones formales en las que todo está decidido y en los que se evidencian relaciones excesivamente jerárquicas entre docentes, en su papel de expertos, y las familias. En consecuencia, se confunde la presencia de las familias en el órgano de toma de decisiones con su implicación y elección de los asuntos.

- Otros espacios de participación

Más allá de la participación que acontece en espacios formalmente dispuestos, encontramos acepciones de participación que buscan en las familias colaboradores para la propuesta educativa definida por los docentes, pero no codecisores (Fernández-Enguita, 1993). Así, se promueven actividades que implican a las familias en las actividades de aprendizaje de sus hijos a través de acciones altamente estructuradas por el profesorado: "el día de (las profesiones, los países, etc.)", la maleta viajera o la búsqueda de información en casa de los temas de trabajo del aula. Si bien la búsqueda de nuevos espacios de implicación de las 
familias debe ser celebrada, reducir su presencia a estas actividades simbólicas no amplía su agencia como agentes educativos.

Consecuentemente, es imprescindible reflexionar sobre los espacios de participación existentes (Ellis y Hughes, 2002; Mora, 2002) y sobre cómo ampliarlos desde la consideración de que la participación implica tener un poder compartido (Lodge, 2005) y supone generar lugares de encuentro, diálogo, argumentación y decisión participada.

\section{Conclusiones}

Este trabajo busca examinar las comprensiones acerca de los procesos de participación familiar en las escuelas que se manejan en los diferentes estudios, investigaciones y normativas existentes. Entendemos que organizar de manera sistemática dichas conceptualizaciones y las acciones derivadas permite reflexionar sobre este ámbito de la vida escolar $y$, en un momento subsiguiente, encaminar significados y actuaciones comprometidas con los objetivos inclusivos y democráticos con los que se alinea este trabajo (Apple y Beane, 2005; Bolívar, 2006; Fielding y Moss, 2011).

A partir de los hallazgos conseguidos, tratamos de enunciar de manera esquemática algunas conclusiones que pueden contribuir a mejorar los procesos de participación de las familias en la escuela desde la lógica democrática que pretendemos.

Respecto a su involucración en los procesos de enseñanza-aprendizaje, observamos que las normativas promocionan únicamente procesos comunicativos, normalmente unidireccionales (de escuela a familias), sobre los progresos y dificultades del alumnado en el aprendizaje, especialmente en el momento de la evaluación. Sin embargo, desde los lineamientos democráticos, se precisa incluir otras acciones que favorezcan una incidencia relevante de las familias en el currículum:

- Acceso de las familias al conocimiento de la vida escolar desde la transparencia, utilizando códigos lingüísticos accesibles y compartidos.

- Intercambio de información bidireccional, analizando colaborativamente y tomando decisiones de manera conjunta (López, Ridao y Sánchez, 2004).

- Ampliar los espacios de toma de decisiones a aspectos relevantes 
de la vida escolar, como el currículum, deliberando juntos sobre objetivos, actividades, modos de evaluación y cualquier otro elemento curricular (Apple y Beane, 2005; Fielding y Moss, 2011).

Otro de nuestros hallazgos tiene que ver con el papel de las familias en la atención a la diversidad, que permite inferir, de otra parte, las comprensiones acerca de esta cuestión. Así, encontramos una interpretación de diversidad como problema que hay que enfrentar y solventar, desde procesos de evaluación y medidas donde las familias deben estar informadas y consentir únicamente. Sin embargo, aproximarnos a los principios inclusivos conlleva transformar esta cultura y sus prácticas:

- Supone reconocer que diversidad no se identifica como un problema, sino con la heterogeneidad de intereses, necesidades, conocimiento, valores, lenguajes o contextos que todos los estudiantes tienen.

- Consecuentemente, implica considerar el conocimiento privilegiado que tienen las familias $y$, por ende, entender que pueden ser agentes de análisis muy valiosos que permitan pensar en otra escuela que acoja, de la bienvenida y promueva el aprendizaje de todo el alumnado.

Finalmente, observamos que tanto los espacios formales como informales de participación se restringen a la toma de decisiones sobre asuntos secundarios en la vida en la escuela (festividades, actividades extraescolares y complementarias, etc.). Estos hallazgos enlazan con el modelo profesional técnico reinante que considera al docente como experto y único agente facultado para decidir sobre cuestiones que requieren experticia, como los asuntos organizativos y curriculares. En tal sentido, es necesario:

- Poner en valor argumentos que defiendan la involucración de las familias en la escuela como derecho que brota de su condición de padres, pero también de ciudadanos que pueden participar en un espacio de naturaleza pública.

- Sanear los canales de participación existentes. Bolívar (2006) define algunas líneas de actuación: promover iniciativas de comunicación que tengan una orientación dialógica y que permitan participar en cuestiones educativas centrales, como el campo de lo curricular; participar en la configuración del proyecto educativo 
de centro, entendido como momento clave para esbozar líneas de actuación compartidas y amparar un proyecto pedagógico con el que todos puedan sentirse comprometidos; prestación de servicios comunitarios al centro, donde el AMPA puede convertirse en elemento clave para desarrollar acciones junto con los municipios.

\section{Referencias}

Aguilar, M., y Leiva, J., (2012). La participación de las familias en las escuelas TIC: análisis y reflexiones educativas. Pixel-Bit: Revista de medios y educación, 40, 7-19.

Álvarez, M. (2017). Hacia un modelo integrador de la tutoría en los diferentes niveles educativos. Educatio Siglo XXI, 35, 21-42.

Álvarez, I., Campos, M., Carmona, P., Magan, E., Martínez, I., y Sánchez, C. (2014). La familia en la escuela: aprender haciendo... Aula de infantil, 75, 38-40.

Angulo, J. F. (1994). Teoría y desarrollo del curriculum. Málaga, España: Aljibe.

Angulo, A. (2009). La tutoría en la educación primaria: manual de ayuda. Barcelona, España: Wolters Kluwer.

Apple, M., y Beane, J. (2005). Escuelas democráticas. Madrid, España: Morata.

Arostegui, I., Darretxe, J., y Beloki, N. (2013). La participación de las familias y de otros miembros de la comunidad como estrategia de éxito en las escuelas. Revista Iberoamericana de Evaluación Educativa, 6(2), 187-200.

Atlántida, P. (2003). Escuelas y familias democráticas Proyecto Atlántida. Recuperado de: http://www.proyectoatlantida.eu/wordpress/wp-content/uploads/2015/01/Escuelasy-Familias-Democr\%C3\%A1 ticas.pdf

Auduc, J. (2007). Les relations parents-enseignants à l'école primaire. París, Francia: CRDP de I'académie de Créteil.

Bastiani, J. (1987). Parents and teachers. Perspectives on home-school relationes. Nelson, EEUU: Windsor.

Besalú, C. (2010). Mejorar la relación entre los centros escolares y las familias del alumnado. En J. Lara, Colectivo Amani, L. Ten, R. Flecha, X. Besalú (coord.). Libro blanco de la educación intercultural (155-156). Recuperado de: http://aulaintercultural. org/2010/06/23/libro-blanco-de-la-educacion-intercultural/

Bolívar, A. (2006). Familia y escuela: dos mundos Ilamados a trabajar en común. Revista de educación, 339, 119-146.

Boza, Á., Salas, M., Ipland, J., Aguaded, M. C. y Fondón, M. (2005). Ser profesor, ser tutor. Orientación Educativa para Docentes. Huelva: Hergué.

Cánovas, P., y Sahuquillo, P. (2012). La cooperación tutorial con la familia y la comunidad. Valencia, España: Nau Llibres.

Castro, A., y García, R. (2016). Vínculos entre familia y escuela: visión de los maestros en formación. Magis, Revista Internacional de Investigación en Educación, 9(18), 193-208.

Ceballos, N. y Saiz, Á (en prensa). La acción tutorial como escenario de colaboración familia-escuela. Revista española de orientación y psicopedagogía. 
Un proyecto educativo común: la relación familia y escuela. Revisión de investigaciones y normativas

Noelia Ceballos lópez y Ángela Saiz Linares

Corpas, C., Ramírez, A., Mérida, R. y González, E. (2012). La coordinación del profesorado como estrategia para innovar en la docencia concretada en "Orientación Educativa: Escuela, Familia y Comunidad" en la titulación de grado de Educación Infantil. Recuperado de: http://riemann.upo.es/congresos/index.php/innovagogia2012/ linnovagogia2012/paper/viewFile /74/76

Comisión Europea (2000). Informe Europeo sobre la Calidad de la Educación Escolar. Recuperado de: http://www.parentsparticipation.eu/sites/default/files/pagina/6._comision_eu_2000.pdf

Creswell, J. (1994). Research design qualitative and quantitative approaches. Thousand Oaks, EEUU: Sage Publications.

Decreto 25/2010 de 31 de marzo por el que se aprueba el Reglamento Orgánico de las Escuelas Infantiles, de los Colegios de Educación Primaria y de los Colegios de Educación Infantil y Primaria en el ámbito territorial de la Comunidad Autónoma de Cantabria.

Decreto 98/2005, de 18 de agosto, de ordenación de la atención a la diversidad en las enseñanzas escolares y la educación preescolar en Cantabria.

Consejo Escolar Del Estado (2014). La participación de las familias en la educación escolar. Madrid, España: Ministerio de Educación, Cultura y Deporte.

Eccles, J., y Harold, R. (1996). Family involvement in children's and adolescents' schooling. En A. BOOTH, y J. DUNN (eds.), Family school links: how do they affect educational outcomes? (pp. 3-34.). New Jersey: Erlbaum

Egido, I. (2014). Marcos normativos de la participación de las familias en los sistemas educativos europeos. Una visión comparada. En Consejo Escolar del Estado, La participación de las familias en la educación escolar (pp. 35-56). Madrid, España: Ministerio de Educación, Cultura y Deporte.

Ellis, D., y Hughes, K. (2002). Partnerships by Design: Cultivating Effective and Meaningful School-Family-Community Partnerships. Creating Communities of Learning \& Excellence. Portland, OR: Northwest Regional Educational Laboratory.

Epstein, J. L. (2001). School, family and community partnerships. Preparing educators and improving schools. Colorado, EEUU: Westview Press.

Feito, R. (2014). Treinta años de consejos escolares. La participación de los padres y de las madres en el control y gestión de los centros sostenidos con fondos públicos en España. Revista de currículum y formación del profesorado, 18(2), 51-67.

Fernández-Enguita, M. (1993). La profesión docente y la comunidad escolar: Crónica de un desencuentro. Madrid, España: Morata.

Fernández-Prada, F. (2003). Participación de padres y alumnos, ¿imposición, moda o reto?. En AAVV. La participación de los padres y madres en la escuela (pp. 45-54). Barcelona, España: Graó,

Fielding, M., y Moss, P. (2011). Radical education and the common school: A democratic alternative. London, UK: Routledge.

García-Bacete, F. J. (2003). Las relaciones escuela-familia: un reto educativo. Infancia y aprendizaje, 26(4), 425-437.

García-Albaladejo, A. (2006). Participación de las familias en la vida escolar: acciones y estrategias. Madrid, España: Ministerio de Educación y Ciencia. 
Garreta, J. (2008). La participación de los padres en la escuela pública. Las asociaciones de madres y padres del alumnado. Madrid, España: CEAPA.

Garreta, J. (2010). La participación de las familias en la escuela. En R. Feito (coord.). Sociología de la educación secundaria (pp. 47-66). Barcelona, España: Graó.

Garreta, J. (2013). La participación de las familias en la escuela: una cuestión pendiente. Documentación Social, 171,101-124.

Garreta, J. (2015). La comunicación familia-escuela en educación infantil y primaria. RASE: Revista de la Asociación de Sociología de la Educación, 8(1), 71-85.

Giró, J., Mata, A., Vallespir, J., y Vigo, B. (2014). Familias y escuelas: los diferentes discursos sobre la participación. Ehquidad International Welfare Policies and Social Work Journal, 2, 65-90.

Hernández, M., y López, H. (2006). Análisis del enfoque actual de la cooperación padres y escuela. Aula abierta, 87, 3-26.

Issó, D., Becerril, D., y Venegas, M. (2019). Ojalá mi madre hubiera hecho por mí lo que yo estoy haciendo por mi hija. Sociologia, Problemas e Práticas, 89, 33-52.

Kim, J., y Bryan, J. (2017). A first step to a conceptual framework of parent empowerment: Exploring relationships between parent empowerment and academic performance in a national sample. Journal of Counseling \& Development, 95(2), 168-179.

Kohl, G., Lengua, L., y Mcmahom, R. (2000) Parent involvement in school. Conceptualizing multiple dimensions and their relations with family and demographic risk factors, Journal of School Psychology, 38(6), 501-523.

Ley Orgánica 8/2013, de 9 de diciembre, para la mejora de la calidad educativa (LOMCE).

Llevot, N., y Bernad, O. (2015). La participación de las familias en la escuela: factores clave. RASE: Revista de la Asociación de Sociología de la Educación, 8(1), 57-70.

Lodge, C. (2005). From hearing voices to engaging in dialogue: problematising student participation in school improvement. Journal of Educational Change, 6, 125-146.

Longás, J., y Molla, N. (2007). La escuela orientadora. La acción tutorial desde una perspectiva institucional. Madrid: Editorial Narcea.

López-Noguero, F. (2002). El análisis de contenido como método de investigación. Enclave pedagógica, 4, 167-180.

López, I., Ridao, P., y Sánchez, J. (2004). Las familias y las escuelas: una reflexión acerca de entornos educativos compartidos. Revista de Educación, 334, 143-163.

Macià, M. (2016). La comunicación familia escuela: el uso de las TIC en los centros de primaria. Revista Electrónica Interuniversitaria de Formación del Profesorado, 19(1), 73-83.

Mata, A. (2016). Evolución de la participación de las familias en el sistema escolar español. Intercambio/Échange, 1, 181-194.

MacBeth, A. (1984). The child bethween. A repport on school-family relations in the countries of the European Community. Bruselas: Office Publications of the European Communities.

Maestre, A. (2009). Familia y escuela. Los pilares de la educación. Revista digital Innovación y experiencias educativas, 14, 1-11.

Mendes, R., y Miskulin, R. (2017). Content analysis as a methodology. Cadernos de Pesquisa, 47(165), 1044-1066. 
Un proyecto educativo común: la relación familia y escuela. Revisión de investigaciones y normativas

Noelia Ceballos lópez y Ángela Saiz Linares

Méndez, L., Ruiz, J., Rodríguez, E. y Rebaque, M. O. (2010). La tutoría en educación infantil. Madrid, España: Wolters Kluwer.

Mora, J. (2002). Canales de comunicación familia-escuela. Infancia: educar de 0 a 6 años, 74, 10-14.

Ocampo, C., Caeiro, M., y Sarmiento, J. (2012). Las tic y la función tutorial en la etapa de educación infantil: la opinión del profesorado de centros públicos del sur de Galicia. Revista española de Orientación y Psicopedagogía, 23(2), 60-78.

Orden EDU/105/2008, de 4 de diciembre, por la que se regula la evaluación de la educación Infantil en la Comunidad Autónoma de Cantabria.

Orden EDU/65/2010, de 12 de agosto, que aprueba las instrucciones que regulan la organización y funcionamiento de las escuelas infantiles, de los colegios de educación primaria y de los colegios de educación infantil y primaria de la Comunidad Autónoma de Cantabria.

Pantoja, A. (2013). La acción tutorial en la escuela. Madrid, España: Síntesis

Pastor, M. (2009). La acción tutorial en educación infantil y. Primaria. Revista digital de innovacción y expereincias educativas, 16, 1-12.

Paterman, C. (1970). Paticipation and democratic theory. London, UK: Cambridge University Press.

Pugh, G., y De'ath, E. (1989). Towards Partnership in the Early Years. London, UK: National Children's Bureau.

Reininger, T., y Santana López, A. (2017). Parental involvement in municipal schools in Chile: Why do parents choose to get involved?. School Psychology International, 38(4), 363-379.

Ruiz-Silva, A. (2004). Texto, testimonio y metatexto. El análisis de contenido en la investigación en educación. En A. Jiménez y A. Torres (Comps.), La práctica investigativa en Ciencias Sociales (pp.45-61). Bogotá, Colombia: Universidad Pedagogica Nacional.

Santos-Guerra, M. Á. (2000). La participación es un árbol: padres y madres, desde la ciudadanía, hacen la escuela. Kikiriki. Cooperación Educativa, 55, 105-116.

Saracostti, M., Lara, L., Martella, D., Miranda, H., Miranda-Zapata, E.D. y Reininger, T. (2019). Influence of family involvement and children's socioemotional development on the learning outcomes of Chilean students. Frontiers in Psychology, 10(335), 1-7.

Sarramona, J., y Rodríguez, T. (2010). Participación y calidad de la educación. Aula Abierta, 38(1), 3-14.

Slee, R. (2011). The irregular school: Exclusion, schooling and inclusive education. United Kingdom: Taylor \& Francis.

Susinos, T., y Ceballos, N. (2012). Voz del alumnado y presencia participativa en la vida escolar. Apuntes para una cartografía de la voz del alumnado en la mejora educativa. Revista de Educación, 359, 24-44.

Urosa, B., y Lázaro, S. (2017). La función tutorial en educación infantil y primaria: actividades que implica y dificultad percibida por el profesorado en su desarrollo. Educatio Siglo XXI, 35, 111-138. 